\title{
Root Dentine Translucency of Human Teeth: Factors Affecting Formation and Deposition
}

\author{
${ }^{1}$ L. Vasiliadis, ${ }^{1}$ C. Stavrianos, ${ }^{2}$ P. Dagkalis, ${ }^{1}$ Ks. Parisi, ${ }^{1} \mathrm{O}$. Pantelidou and ${ }^{1}$ E. Samara \\ ${ }^{1}$ Department of Endodontology (Forensic odontology), School of Dentistry, \\ Aristotle University, Thessaloniki, Greece \\ ${ }^{2}$ Department of Preventive Dentistry Periodontology and Implant Biology, \\ School of Dentistry, Aristotle University, Thessaloniki, Greece
}

\begin{abstract}
Aim of the present study is to indicate the factors contributing in changes of the formation and deposition of root dentine which results in translucency of the root dentine. The root dentine translucency of human teeth is already proved to be a significant process in age estimation, since the creation of sclerotic dentine is strictly related with the aging of the individual. Different factors seem to affect this deposition with the environmental, one playing the most significant extraneous factor, changing the rate and type of creation of translucent root dentine. The mechanical factors are analyzed in order to imprint all the factors that may interfere with the deposition process and as a result in the proper age estimation.
\end{abstract}

Key words: Dentine translucency, age determination, endodontology, Forensic odontology, extraneous factor, Greece

\section{INTRODUCTION}

Dentine translucency occurs by the scattered light inside the dentinal tubules due to its contents. Opacity is present in zones of translucent dentine which depicts the time of deposition. As it has been shown by numerous studies, there is forensic use of this biological characteristic. Not only the eruption status but also the developmental state of the tooth can define the age of the individual (Sengupta et al., 1999). This can be done by the research process of the root dentine translucency as its formation is strongly related with the individual's age. Translucency of dentine is the optical manifestation of changes in the dentine. The only proof of age estimation is the optical translucency that is optically recognized. As it has been shown the dentine in the areas of high mineralization allows the light to pass through it and does not reflect and scatter light as normal dentine does. Breakthrough in the discovery and further investigation was the use of Scanning Electron Microscopy (SEM) in the examination of dentine root translucency. SEM gives the opportunity to study structures about $0.2 \mu \mathrm{m}$ in size. This gives a great advantage to regard the organic fractions and mineral crystals that cause the opacity of the aging root (Vasiliadis et al., 1983, 2008, 2009). Age estimation as it is very important in medicolegal cases for establishing part of the identification process, needs a variety of methods to be confirmed. In addition, ethnic, humanitarian, archeological and demographic reasons intrude a sufficient age estimation of individuals (Harris et al., 2010). Thus, the dentine root translucency becomes an important process in age estimation (Sengupta et al., 1999). Apart from all these stated above, the translucency of the dentine implies in certain biological rules that alters its formation and deposition. Factors such as the environment play a role in the final form of mineralization according to age. Some factors have a more significant outcome than others and some do not seem to intervene in this process (Vasiliadis, 1981).

Factors leading to the translucency of the root dentine: There seems to be no doubt that the reason the root dentine appears translucent is the occlusion of the tubules by calcific material which has a refractive index, very close to that of the rest of the dentine. Despite this, there is a fundamental disagreement between most workers concerning the factors which result in the occlusion of the tubules of the root dentine, whilst the tubules in other areas of the dentine are not occluded and consequently remain opaque.

As stated previously, the relationship between translucency of the root dentine and increasing age was shown during the 1940's and used as one of the criteria for age determination from human teeth by Gustafson

Corresponding Author: Stavrianos Christos, Department of Endodontology (Forensic odontology), Aristotle University, Thessaloniki, Greece 
(1950). This led to the belief that he root dentine translucency is not only associated with age but arises strictly as a result of increasing age. This was challenged by Shroff (1952) who states that since the odontoblasts of the root dentine are chronologically younger than those of the crown dentine, it is unlikely that they would be the first to age. He believed that the true reason for this condition is not increasing age but the different stimuli to which the two groups of odontoblasts are subjected. The odontoblasts of the crown are stimulated by positive pressure transmitted to them by way of the enamel, whereas the odontoblasts of the root are stimulated by negative pressure by way of tension from the cementum and periodontal fibres. Negative pressure results in inactivity and partial atrophy of the odontoblasts lining, the root canals and atrophy of their processes which at a later stage become sclerosed. Shroff's hypothesis however, contradicts findings proving that the pattern of translucency in the dentine of unerupted teeth corresponds to that of erupted teeth. If Shroff is correct then since no stimuli of the above kind are received by the odontoblasts of unerupted teeth, all the dentine including the coronal part should become equally translucent. Bradford $(1958,1967)$ postulates that there is a slow but steady deposition of peritubular dentine on the walls of the dentinal tubules which leads ultimately to obliteration of the lumen. The closure of the tubules should therefore occur first in the narrowest tubules, i.e., the tubules of the root apex (and the terminal branches for that matter) and proceed coronally occluding the larger tubules with increasing age. Contrary to Shroff (1952), Bradford $(1958,1967)$ postulates that the occlusion of the tubules of the root dentine is the result of the normal function of the odontoblasts.

Present knowledge about the dentinal tubules in the crown and root dentine as well as the majority of microradiographic and SEM work done on this topic, supports Bradford's explanation. This seems to show that the material occluding the tubules is mineralized to the same degree as peritubular dentine and is continuous with it. Studies by TEM however, show differences between peritubular dentine and the occluding material, differences in the development of these substances and also show some physiologically occluded tubules in areas where peritubular dentine is absent (Tronstad, 1973a). Also, the disappearance of the odontoblasts and the predentine layer under the translucent root dentine observed by Miles $(1961,1972)$ as well as observations on the odontoblasts of the root dentine (Selvig, 1968) are findings which indicate that the normal reaction of the odontoblasts of the root may not occur in the manner argued by Bradford. Furthermore, histological studies on human teeth suggest that the part of the odontoblastic process thought to be related to the formation of peritubular dentine lies more pulpally than the zone where occlusion of the tubule takes place (Symons, 1967a). Findings of electron microscopists (Brannstrom and Garberoglio, 1972; Garberoglio and Brannstrom, 1976; Holland, 1975, 1976a, b) indicate that the odontoblastic processes are not present in the peripheral parts of the lumens. These observations do not support Branford's explanation, since the occluding material as opposed to peritubular dentine is not deposited directly by the odontoblast process.

Miles $(1961,1972)$ appears to be unconvinced that root dentine translucency is an age change independent of environmental influences. He suggests that it is more plausible that this condition is related to periodontal disease and is due to the reaction of odontoblasts to bacterial toxins derived from the gingival margin. $\mathrm{He}$ postulates that some degree of slowly progressive periodontal disease is almost universal and that the process starts in young adult life. This would explain the apparent correlation of apical translucency with age. He argues that the toxins reach the apical part of the pulp as a result of the pumping action of tooth movement and that occasional pumping action of greater force would carry toxins further into the pulp. This would tent to produce a gradually extending effect because, like all random events, it would tend to accumulate with time. He postulates that in the main, the toxins must act directly on the body of the odontoblasts and not on the peripheral end of the tubules because otherwise since, the final result is the occlusion of the tubules, the procedure would be self-limiting. Miles' main supporting evidence is the observed disappearance of the predentine layer and the odontoblasts under the translucent root dentine which he believes shows that the changes of the translucency in apical dentine are associated with the ultimate disappearance of the odontoblasts. The previously mentioned findings, however that the root dentine in unerupted teeth becomes translucent in a similar way to that of erupted teeth make Miles' explanation untenable as toxins from the periodontal tissues could not affect unerupted teeth.

\section{RESULTS AND DISCUSSION}

Under normal circumstances, there is no doubt that every dentinal tubule passing through the predentine corresponds to one odontoblast. Ultrastructural studies of human dentine performed with TEM have shown that at least in the predentine and in the dentine adjacent to the predentine, the odontoblastic process completely fills 
the lumen of the dentinal tubules (Shroff, 1952; Arwill and Bloom, 1955; Frank, 1959; Awazawa, 1962; Arwill, 1967; Brannstrom and Garberoglio, 1972; Jenkins, 1978). The structural relationship of the odontoblast process to the wall of the dentinal canal is of interest in relation to the circulation of tissue fluid within the dentine. For continued mineralization of the tissue and sclerosis of dentinal canals to take place, movement of electrolytes to the peripheral areas of dentine is necessary. This could be achieved without difficulty if a space existed between the plasma membrane of the odontoblast process and the wall of the lumen. However, if the odontoblast completely fills the lumen as the above studies show extra cellular circulation would be minimal. An intracellular transport mechanism for the elements of the mineral component must be considered (Johansen and Parks, 1962; Johansen, 1967).

This tissue fluid as manifested by the formation of pulp stones and pulpal calcifications in general, contains a sufficient amount of minerals to promote calcification. Consequently, the additional calcification of the dentine that takes place behind the calcification front of the normal dentine depends entirely upon the minerals brought to that site by the odontoblasts through their processes. The deposition of these minerals is highly organized and results in an increase in the mineralization of the already highly mineralized intertubular dentine (Amprino and Camanni, 1956; Takuma, 1960; Miller et al., 1971; Tronstad, 1973b; Jenkins, 1978) and in the formation of the peritubular dentine (Arwill and Bloom, 1955; Blake, 1958; Atkinson and Harcourt, 1961; Harcourt, 1964; Frank, 1968). If for any reason, the sealing of the pulpal end of the lumens of the tubules by the odontoblastic processes is broken then there would be no reason why the fluid in the pulp chamber and the root canal cannot enter the dentinal tubules.

The organic matrix (Takuma, 1960; Johansen and Parks, 1962; Frank, 1968; Boyde and Lester, 1967; Mjor, 1972; Shimauchi et al., 1973) and the apatite crystals existing on the surface of the tubule wall act as mineralization seeds. Experiments performed in vitro under conditions as similar as possible to normal physiological conditions (i.e., $\mathrm{pH}$ around 7 , temperature $37^{\circ} \mathrm{C}$ and with the concentration and types of minerals in the experimental fluids identical with physiological fl uid) show that pieces of human dentine and predentine do act as seeds for the precipitation of the minerals and do calcify (Solomons and Neuman, 1960; Tomazic and Nancollas, 1975; Tomson et al., 1977). Furthermore, the in vitro experiment has shown that the degree of mineralization of the pieces of the dentine and predentine used as seeds increased with time. The findings of previous researchers indicate that this may be the procedure by which the dentinal tubules of the human root dentine become occluded and consequently, the corresponding areas of the dentine appear translucent. These findings are; translucency of previously normal dentine is the result of mineral deposition in the lumens of the dentinal tubules with a in gradual reduction of the diameter of the lumen of the tubules, eventually resulting in the complete obliteration of the lumen. These mineral deposits have a refractive index similar to that of the rest of the dentine.

The absolute increase in the amount of minerals in the zones of the translucent dentine proves that the occluding minerals are not provided by the adjacent inter-tubular dentine but orginate from another source. This other source can only be the pulp chamber and root canal since if it arose from an external source and passed through the cementum, the process of translucency would be self-limiting. Furthermore, the finding that the amount of translucency in non-vital teeth is well below that of vital teeth of corresponding ages supports this conclusion (Bang and Ramm, 1970). Consequently, the maintenance of the blood supply of the pulp is necessary for the appearance of translucency in the root dentine since, it represents the source of minerals.

Both TEM and SEM have shown that the occluding material is eventually almost or completely indistinguishable from peritubular dentine. However according to present knowledge, the formation of peritubular dentine is governed by the odontoblast and its process. These techniques have shown that the process of development and formation of the occluding material is fundamentally different from that of the peritubular dentine which proceeds in an appositional fashion maintaining a clear forming front whereas the occlusive changes occur rather diffusely (Takuma and Eda, 1966) with the packing of the occluding minerals increasing in stages with time. This process apparently occurs without the necessity for a vital odontoblast (Furseth, 1974; Holland, 1976b). Occlusion of the tubules occurs despite irregularities in the development of the peritubular dentine (Tronstad, 1973b) and in areas where peritubular dentine is absent, i.e., interglobular spaces (Tsatsas and Frank, 1972). Symons (1967b) states that if the conclusion from histo-chemical studies that the more reactive, pulpal and part of the odontoblastic process is related to the formation of the peritubular dentine is correct then it may be that occiusion of the dentinal tubules in a fully formed tooth is not produced by further deposition of peritubular dentine proper but may be brought about by a less organised calcific deposit. The finding by SEM of spherical bodies in the lumen of the tubules, identical with those found by previous researchers (Lester and Boyde, 1968; Brannstrom and 


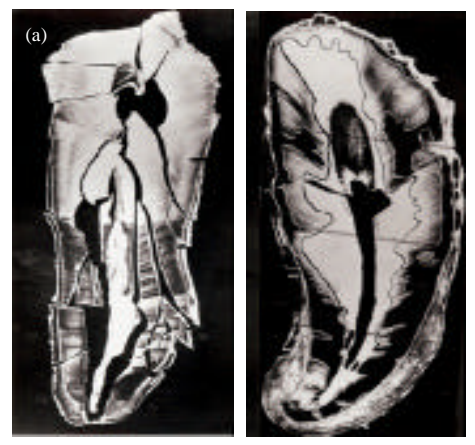

Fig. 1: a) Fragments of a section (in air) photographed before mounting on stubs for SEM examination in order to record the relationship between translucent areas of dentine and fractured edges (mag. X 8.0). Male, age 66, lower right canine; b) section illustrating translucent (black), normal (white) and semi-translucent (grey) areas of dentine, (mag. X 13.0). Female, age 67, lower left canine, unerupted (Vasiliadis, 1981)

Garberoglio, 1972) is another developmental difference between the occiuding material and the peritubular dentine. Based on the appearance and the locations of these round bodies, the above researchers conclude that such outgrowth may represent the first stage in the occlusion of the tubules with material of the same nature as the peritubular dentine. The next stage of the occlusion of the tubules would be the confluence of these spherical bodies and thus the formation of the occluding rods. More impressive evidence is provided by the appearances of these bodies and that of the material occluding the tubules as it appears in specimens fractured transversely to the long axis of the tubules (Fig. 1) which are identical with the appearances of the minerals formed during in vitro experiments in which human dentine and predentine were used as seeds for mineralization (Tomson et al., 1977).

Finally, the slight annular spaces occasionally observed between peritubular dentine and occluding material under SEM, support the conclusion that although peritubular dentine and occluding material are of the same nature, their development is fundamentally different. In conclusion, it must be emphasized that the pattern of deposition of the occluding minerals appears similar to that of the in vitro calcification of dentine in both cases, the packing of the mineral increases with time and there is a striking similarity between the appearance of the occluding material during different stages of mineralization and that of the in vitro calcifications. All investigators who have studied the age changes of the cervical and coronal part of the human pulp report a reduction in the number of the sub-odontoblastic pulpal blood vessels with increasing age (Kramer, 1960; Zerlotti, 1964; Bennett et al., 1965). Also, they report a reduction in the number of the odontoblasts (Zerlotti, 1964; Symons, 1967a, b). Despite the reduction of the numbers of the odontoblasts and their blood supply with age, the corresponding part of the dentine does become translucent with age and furthermore at an increasing rate. This was shown and by those of Miles (1963), Johanson (1971), Azaz et al. (1977) and Vasiliadis (1981). Apparently therefore, the presence of odontoblasts is not necessary for the development of translucency. Miles (1963) reports that no predentine and odontoblasts are present at the pulpal end of the translucent zones of human root dentine. The important question is whether the translucency is the result of the disappearance of the odontoblasts or the disappearance of the odontoblasts the result of the changes that also caused the appearance of the translucency. Miles $(1963,1976)$, reasoning that periodontal toxins by affecting the corresponding odontoblasts result in the appearance of the translucency, believes that changes in the odontoblasts precede the changes responsible for the appearance of the translucency. According to Vasiliadis (1981) and also those of most previous researches, support the explanation that translucency arises after the changes in the odontoblasts. These findings are; those which showed that the odontoblasts apparently played no role in the formation of the occluding material; the pattern of distribution of translucency in the root dentine.

Areas of the pulpal surfaces of the teeth from which predentine and calcospherites were absent but on which patent tubule openings were found. The onset of the changes of the pulpal surfaces appears considerably before the appearance of translucency in the corresponding areas of the root dentine.

That the number of the dentinal tubules per unit area is drastically reduced in the circumpulpal dentine (Kramer, 1951) and in the mineralizing front of the apica one third of the root as compared with that of corresponding but more coronal dentine (Whittaker and Kneale, 1979).

The findings of Seelig (1965) who studied $\mathrm{H}$ and $\mathrm{E}$ stained sections of dentine of 250 decalcified human teeth. Seelig observed atrophy and death of groups of odontoblasts of the apical region of human teeth from patients of different ages. This process commences only 1 or 2 years after full eruption of the tooth and extends coronally in the same fashion with age. The areas of pulpal dentine surface corresponding to the lost odontoblasts were devoid of a predentine layer. Summarizing his findings, Seelig (1965) states that there is a loss of odontoblasts which die in clumps in areas in which there is no crowding. The loss of odontoblasts 
then proceeds upward from the apex of the roots, generally to a greater extent in older individuals. This loss of odontoblasts occurs early in the development of the tooth, independent of local pathological changes in the dentine. The formation of tubular secondary dentine is concomitant to the loss of the odontoblasts and in each succeeding layer, the dentine has fewer tubules.

Mjor and Furseth (1968) and Furseth and Mjor (1972) examined the dentine and pulp under corticosteroid-lined experimental cavities prepared in intact human young premolars. The cavities were prepared in vivo. The teeth were then extracted after observation periods varying from 14-38 days and processed for TEM. They found in teeth with observation periods $>20$ days a localized marked reduction or absence of the predentine layer corresponding to the areas of the experimental cavities. Since, adjacent areas had normal predentine, they conclude that the predentine layer present before the experimental cavities mineralized during the 1 st 20 days after the experiment and no further production of predentine took place. They also found that odontoblastic layer corresponding to the cavities was markedly reduced. The odontoblasts were reduced both in size and number. The typical palisade-like arrangement of the odontoblasts was lost in these areas. They give convincing evidence that the corticosteroid lining of the cavities and not the preparation of the cavities was responsible for these changes, i.e., by comparison with the changes produced under similarly prepared cavities unlined by corticosteroids and with similarly prepared cavities lined by a material with very low corticosteroid concentration. Although, they found no difference in the intertubular areas, they found some of the tubules below the prepared cavities, partly or completely obliterated. The partly obturated tubules showed eccentric growth of mineral tissue. The electron density of the obturating material was in these cases, similar to that found in the peritubular matrix. It should be emphasized that these electron dense obturations in corticosteroid-covered dentine were found in an area of the dentine where no highly mineralized peritubular matrix is normally found. A structurally similar type of obturation of dentinal tubules to that found in corticosteroid-covered dentine has also been demonstrated in old sclerotic root dentine.

Apparently, the atrophy and disappearance of some of the odontoblasts initiates the mechanisms that cause the corresponding root dentine to become translucent. This process does not appear to be the result of local or generalized pathological conditions external irritations and crowding of odontoblasts. Furthermore, this disappearance of the odontoblasts of the root prevents the coronal part of the pulp from becoming strangled. The disappearance of the odontoblasts of the root may well be a genetically programmed stage of tooth development. Translucency appears to be a result of the disappearance of these odontoblasts. This is very fortunate, since the crown is the part of the tooth usually subject to external irritations and the response of the pulpo-dentinal complex to irritations may be vital for the survival of the tooth.

\section{CONCLUSION}

Bodecker (1925), Gustafson (1950) and others to follow have thoroughly researched the age estimation process via root transparency. This procedure starts with the extraction of the tooth, section and polish before examination (Harris et al., 2010). Sognnaes et al. (1985) proposed a method of measuring the sclerotic dentine in intact teeth. The gradual progressive sclerosis of the tubules from the apex to the coronal creates the root transparency (Vasiliadis, 1981; Tziafas, 1999). The dentinal tubules narrow progressively from $3.2 \mu$ in young individuals to about $1.5 \mu$ at 50 and down to $1.2 \mu$ at 70 years of age.

The changes in dentine usually are spotted at adulthood but may start at late teenage hood (Vasiliadis et al., 1983). Opaqueness of young dentine is due to differences in the refractive indexes between the crystalline and the intratubular organic components. Aging causes the refractive indexes to converge, making the dentine transparent to transmitted light. There may be other reasons for this phenomenon too; toxins for diseased periodontal tissues may form sclerotic dentine, absence of functional stimuli and the diminished diameters of the dentinal tubules due to increased mineralization of the intratubular matrix, especially at the root apex (Whittaker and Bakri, 1996).

The use of methylene blue enhances the contrast between the types of dentine if used in partial vacuum. Also, dietary differences may alter the percentage of sclerotic dentine, affecting less the older adults though (Whittaker and Bakri, 1996).

In certain researches, it has been reported that there is low correlation between the proportion of transparent dentine and chronological age (0.84). The deviation by $>5$ years in the estimation was at least in $55 \%$ of cases (Drusini et al., 1991).

\section{REFERENCES}

Amprino, R. and F. Camanni, 1956. Historadiographic and autoradio-graphic researches on hard dental tissues. Acta Anat. (Basel), 28: 217-258.

Arwill, T. and G. Bloom, 1955. Some remarks on the structure of dentine as revealed by the electron microscope. Acta Odontol. Scand., 12: 185-192. 
Azaz, B., Y. Michaeli and D. Nitzan, 1977. Aging tissues of the roots of nonfunctional human teeth (impacted canines). Oral Surg. Oral Med. Oral Pathol., 43: $572-578$.

Arwill, T., 1967. Studies on the ultra structure of dental tissues. II. The predentine-pulpal border zone. Odontol. Revy, 18: 191-208.

Atkinson, H.F. and J.K. Harcourt, 1961. Some observations on the peritubular translucent zones in human dentine. Aust. Dent. J., 6: 194-197.

Awazawa, Y., 1962. Electron microscope investigation of the dentine with particular regard to the nature of area surrounding the odontoblast process. J. Nihon Univ. Sch. Dent., 5: 31-54.

Bang, G. and E. Ramm, 1970. Determination of age in humans from root dentin transparency. Ácta Odontol. Scand., 28: 3-35.

Bennett, C.G., K.E. Kelln and W.R. Biddington, 1965. Age changes of the vascular pattern of the human dental pulp. Arch Oral Biol., 10: 995-998.

Blake, G.C., 1958. The peritubular translucent zone in dentin. Br. Dent. J., 104: 57-64.

Bodecker, C.E., 1925. A consideration of some of the changes of the teeth from young to old age. Dent. Cosmos, 67: 543-549.

Boyde, A. and K.S. Lester, 1967. The fine structure of peritubular dentine. J. Anat. (London), 102: 144-145.

Bradford, E.W., 1958. The naturation of the dentine. Br. Dent. J., 105: 212-216.

Bradford, E.W., 1967. Microanatomy and Histochemistry of Dentine. In: Structural and Chemical Organization of Teeth, Miles, A.E.W. (Ed.). Academic Press, New York, pp: 3-34.

Brannstrom, M. and R. Garberoglio, 1972. The dentinal tubules and the odontoblastic processes. A scanning electron microscopic study. Acta Odont. Scand., 30: 291-311.

Drusini, A., I. Calliari and A. Volpe, 1991. Root dentine transparency: Age determination of human teeth using computerized densitometric analysis. Am. J. Phys. Anthropol., 85: 25-30.

Frank, R.M., 1959. Electron microscopy of undecalcified sections of human adult dentine. Arch Oral Biol., 1: $29-32$.

Frank, R.M., 1968. Dentinal sclerosis and ultrastructural basis of dentinal sensitivity. Ann. Meet. Am. Inst. Oral Biol., 25: 21-24.

Furseth, R. and I.A. Mjor, 1972. The fine structure of corticosteroid covered, human dentine. Arch. Oral Biol., 17: 719-728.

Furseth, R., 1974. The structure of peripheral root dentin in young human premolars. Eur. J. Oral Sci., 82: $557-561$.
Garberoglio, R. and M. Brannstrom, 1976. Scanning electron microscopic investigation of human dentinal tubules. Archs Oral Biol., 21: 355-362.

Gustafson, G., 1950. Age determinations on teeth. J. Am. Dent. Assoc., 41: 45-54.

Harcourt, J.K., 1964. Further observations on the peritubular translucent zone in human dentine. Aust. Dent. J., 97: 387-392.

Harris, F.E., H.H. Mincer, M.K. Anderson and R.D. Senn, 2010. Age Estimation from Oral andDental Structures. In: Forensic Dentistry, R.D. Senn and G.P. Stimson (Eds.). CRC Press, Taylor and Francis Group, LLC, New York, pp: 285.

Holland, G.R., 1975. The dentinal tubules of the cat and their contents. Ph.D. Thesis, University of Bristol.

Holland, G.R., 1976a. The extent of the odontoblast process in the cat. J. Anat., 121: 133-149.

Holland, G.R., 1976b. An ultra structurant survey of cat dentinal tubules. J. Anat., 122: 1-13.

Jenkins, G.N., 1978. The Physiology and Biochemistry of the Mouth. 4th Edn., Blackwell Scientific Publications, London, UK., ISBN-13: 9780632001385 , pp: 599.

Johansen, E. and H.F. Parks, 1962. Electron microscopic observations on sound human dentine. Archs Oral Biol., 7: 185-193.

Johansen, E., 1967. Ultrastructure of Dentine. In: Structural and Chemical Organization of Teeth, Miles, A.E.W. (Ed.). Academic Press, New York, pp: 35-72.

Johanson, G., 1971. Age determinations from human teeth. Odont. Revy, 21: 1-126.

Kramer, I.R.H., 1951. The distribution of collagen fibrils in the dentin matrix. Br. Dent. J., 91: 1-7.

Kramer, I.R.H., 1960. The vascular architecture of the human dental pulp. Arch Oral Biol., 2: 177-189.

Lester, K.S. and A. Boyde, 1968. The Surface Morphology of Some Crystalline Components of Dentine. In: Dentine and Pulp, their Structure and Reactions, Symon, N.B.B. (Ed.). E and S Livingstone Ltd., Edinburgh and London, pp: 197-205.

Miles, A.E.W., 1961. Ageing in the Teeth and Oral Tissues. In: Structural Aspects of Ageing, Bourne, G.H. (Ed.). Pitman, London, pp: 353-397.

Miles, A.E.W., 1963. Dentition in the estimation of age. J. Dent. Res., 42: 255-263.

Miles, A.E.W., 1972. Sans teeth: Changes in oral tissues with advancing age. Proc. R. Soc. Med., 65: 801-806.

Miles, A.E.W., 1976. Age Changes in Dental Tissues. In: Scientific Foundations of Dentistry, Cohen, B. and I.R.H. Kramer (Eds.). Vol. 29, William Heinemann Medical Books Ltd., London, pp: 363-375.

Miller, W.A., J.D. Eick and M.E. Neiders, 1971. Inorganic components of the peritubular dentin and in young human permanent teeth. Caries Res., 5: 264-278. 
Mjor, I.A. and R. Furseth, 1968. The inorganic phase of calcium hydroxide-and corticosteroid-covered dentine studied by electron microscopy. Arch. Oral Biol., 13: 755-763.

Mjor, I.A., 1972. Human coronal dentine: Structure and reactions. Oral Surg. Oral Med. Oral Pathol., 33: $810-823$.

Solomons, C.C. and W.F. Neuman, 1960. On the mechanisms of calcifi-cation: The remineralization of dentin. J. Biol. Chem., 235: 2502-2506.

Seelig, A., 1965. Dentin and pulp. New York State Dent. J., 31: 54-69.

Selvig, K.A., 1968. Ultra structural changes in human dentine exposed to a weak acid. Arch Oral Biol., 13: 719-734.

Sengupta, A., D.K. Whittaker and R.P. Shellis, 1999. Difficulties in estimating age using root dentine translucency in human teeth of varying antiquity. Arch. Oral. Biol., 44: 889-899.

Shimauchi, K., T. Mashie, S. Fujioka, T. Kizu and K. Ikeda, 1973. A scanning electron microscope study of intratubular dentine fibres. J. Nihon Univ. Sch. Dent., 15: 113-117.

Shroff, F.R., 1952. Thoughts on the physiologic pathology of regressive and reparative changes in the dentine and dental pulp. Oral Surg., 5: 51-58.

Sognnaes, R.F., B.M. Gratt and P.J. Papin, 1985. Biomedical image processing for age measurements of intact teeth. J. Forensic Sci., 30: 1082-1089.

Symons, N.B.B., 1967a. Occlusion of dentinal tubules. J. Dent. Res., 46: 1261-1261.

Symons, N.B.B., 1967b. The Microanatomy and Histochemistry of Dentinogenesis. In: Structural and Chemical Organization of Teeth, Miles, A.E.W. (Ed). Academic Press, New York, pp: 285-324.

Takuma, S. and S. Eda, 1966. Structure and development of the peritubular matrix in dentin. J. Dent. Res., 45: 683-692.

Takuma, S., 1960. Preliminary report on the mineralization of human dentine. J. Dent. Res., 39: 964-972.
Tomazic, B. and G.H. Nancollas, 1975. The seeded growth of calcium phosphates. Surface characterization and the effect of seed material. J. Colloid Interface Sci., 50: 451-461

Tomson, M.B., B. Tomazic, G.H. Nancollas, W. Miller and M. Everett, 1977. The seeded growth of calcium phosphate on dentine and predentin. J. Dent. Res., 56: 1369-1375.

Tronstad, L., 1973a. Quantitative microradiography of intact and worn human coronal dentine. Archs Oral Biol., 18: 533-542.

Tronstad, L., 1973b. Ultrastructural observations of human coronal dentin. Scand. J. Dent., Res., 81: 101-111.

Tsatsas, B.G. and R.M. Frank, 1972. Ultrastructure of the dentimal tubular substances near the dentino-enamel junction. Calcif. Tissue Res., 9: 238-242.

Tziafas, D., 1999. Biology of Dental Tissue. University Studio Press, Thessaloniki, pp: 105-115.

Vasiliadis, L., 1981. Root dentine translucency. Ph.D. Thesis, Bristol University, UK.

Vasiliadis, L., A.I. Darling and B.G. Levers, 1983. The amount and distribution of sclerotic human root dentine. Arch. Oral. Biol., 28: 645-649.

Vasiliadis, L., C. Stavrianos and P. Kafas, 2008. Assessment of dentin translucency in estimating age: A research. Int. J. Orofac. Sci., 1: 4-6.

Vasiliadis, L., C. Stavrianos and P. Kafas, 2009. A forensic aspect of age characteristics of dentine using transversal micro radiography: A case report. Cases J., 2: 4-4.

Whittaker, D.K. and M.J. Kneale, 1979. The dentinepredentine interface in human teeth. A scanning electron microscope study. Br. Dent. J., 146: 43-46.

Whittaker, D.K. and M.M. Bakri, 1996. Racial variations in the extent of tooth root translucency in ageing individuals. Arch. Oral Biol., 41: 15-19.

Zerlotti, E., 1964. Histochemical study of the connective tissue of the dental pulp. Arch Oral Biol., 9: 149-162. 